\title{
ON THE GEOMETRY OF SPHERES IN NORMED LINEAR SPACES
}

\author{
JI GAO and KA-SING LAU
}

(Received 21 January 1988)

Communicated by S. Yamamuro

\begin{abstract}
Some simplifications of Schäffer's girth and perimeter of the unit spheres are introduced. Their general properties are discussed, and they are used to study the $l_{p}, L_{p}$ spaces, uniformly nonsquare spaces, and their isomorphic classes.
\end{abstract}

1980 Mathematics subject classification (Amer. Math. Soc.) (1985 Revision): 46 B 20.

Keywords and phrases: girth, isomorphism, perimeter, uniformly convex, uniformly nonsquare.

\section{Introduction}

Let $X$ be a normed linear space, and let

$$
S(X)=\{x \in X:\|x\|=1\}
$$

be the unit sphere of $X$. In a series of papers, Schäffer made use of the concept of geodesic to study the unit spheres $S(X)$ (see [10] for the complete references). He introduced the following two notations:

$$
m(X)=\inf \{\delta(x,-x): x \in S(X)\},
$$

and

$$
M(X)=\sup \{\delta(x,-x): x \in S(X)\},
$$

where $\delta(x,-x)$ is the shortest length of the arcs joining antipodal points $x$, $-x$ in $S(X)$. He called $2 m(X)$ the girth, and $2 M(X)$ the perimeter of $X$. These parameters, especially $m(X)$, were used to study the $L_{1}$ spaces [8],

(C) 1990 Australian Mathematical Society $0263-6115 / 90 \$ A 2.00+0.00$ 
$C(K)$ spaces [7, 9], reflexivity $[6,11]$ and isomorphism of Banach spaces [10].

Except for the $L_{1}$ spaces, $C(K)$ spaces and Hilbert spaces, the values of the girths and perimeters and difficult to obtain. In [3, 4], Gao considered a simplification of such a concept; he defined the distance of antipodal points $x$ and $-x$ on $S(X)$ as

$$
\alpha(x)=\inf [\max \{\|y-x\|,\|y+x\|\}: y \in S(X)],
$$

and

$$
\begin{aligned}
& g(X)=\inf \{\alpha(x): x \in S(X)\}, \\
& G(X)=\sup \{\alpha(x): x \in S(X)\} .
\end{aligned}
$$

He also defined another function of $x$ and $-x$ on $S(X)$ as

$$
\beta(x)=\sup [\min \{\|y-x\|,\|y+x\|\}: y \in S(X)],
$$

and

$$
\begin{aligned}
& j(X)=\inf \beta(x): x \in S(X)\}, \\
& J(X)=\sup \{\beta(x): x \in S(X)\} .
\end{aligned}
$$

We will call $2 g(X), 2 G(X),(2 j(X), 2 J(X))$ the $\alpha$-girth and $\alpha$-perimeter $(\beta$ girth, $\beta$-perimeter, respectively). Gao then demonstrated the relationships of the above notations, and calculated the values of his girths and perimeters for certain two dimensions spaces, $L_{1}$ spaces and $C(K)$ spaces analogous to Schäffer [10] (for example $g\left(L_{1}\right)=G\left(L_{1}\right)=1, j\left(L_{1}\right)=J\left(L_{1}\right)=2$; $g((C(K))=1$, and $G(C(K))=j((C(K))=J(C(K))=2)$.

In this paper, we will continue the above investigation, we obtain the values of the $\alpha, \beta$-girths and perimeters for $l_{p}, L_{p}$ spaces, and uniformly nonsquare spaces. A relation with the modulus of convexity is also proved. Let the distance of two isomorphic normed linear spaces $X, Y$ be defined as

$$
\Delta(X, Y)=\inf \left\{\ln \|T\| \cdot\left\|T^{-1}\right\|: T: X \rightarrow Y \text { is an isomorphism }\right\},
$$

we will make use of the above girths of $l_{p}, L_{p}$ to obtain bounds for $\Delta\left(l_{p}, X\right)$ (or $\Delta\left(L_{p}, X\right)$ ) so that $X$ is uniformly nonsquare. The application to normal structure will be considered in a forthcoming paper [13].

Our paper is organized as follows. In Section 2, we give some fundamental properties and relationships of the $\alpha, \beta$-girths and perimeters. These concepts will be illustrated by some two dimensional normed linear spaces. Some of the results in this section have appeared in $[3,4]$, we include the simplified proofs here for the sake of completeness and the unavailability of an English translation. 
In Section 3, we find out the $\alpha, \beta$-girths for the special spaces mentioned previously. In Section 4, we use the above girths to study the isomorphic classes of the $l_{p}, L_{p}$ spaces.

\section{Basic properties}

Let $X_{2}$ be a two dimensional linear space. For $x \in S\left(X_{2}\right)$, let $\kappa$ be one of the arcs of $S\left(X_{2}\right)$ from $x$ to $-x$, and let $g:[0, L] \rightarrow \kappa$ be the standard representation in terms of arc lengths, where $L$ is the length of $\kappa$.

LEMMA 2.1. The functions $\phi, \psi:[0, L] \rightarrow[0,2]$ defined by $\phi(s)=\| g(s)-$ $x\|, \psi(s)=\| g(s)+x \|$ are continuously increasing, and decreasing respectively. Moreover, the two curves intersects at only one point.

Proof. The first assertion follows from [10, Theorem 4F]. To prove the second assertion, it is clear that the two curves will meet. We have to show that they intersect at only one point. Assume the contrary, by the monotonicity of $\phi$ and $\psi$, there exists $0<s_{1}<s_{2}<L$ such that

$$
\left\|g\left(s_{i}\right)-x\right\|=\left\|g\left(s_{j}\right)+x\right\| \quad \text { for } i, j=1,2 .
$$

Let $y_{i}, z_{i}$ be the normalization of $g\left(s_{i}\right)-x, g\left(s_{i}\right)+x, i=1,2$, then all these six points are on $\kappa$, and the segments $\left[y_{1} ; y_{2}\right],\left[g\left(s_{1}\right) ; g\left(s_{2}\right)\right],\left[z_{1} ; z_{2}\right]$ are parallel. The convexity of the unit sphere implies that they are collinear and hence $y_{i}=g\left(s_{i}\right)-x, z_{i}=g\left(s_{i}\right)+x, i=1,2$. It follows that

$$
\left\|y_{2}-z_{1}\right\|=2+\left\|g\left(s_{1}\right)-g\left(s_{2}\right)\right\|>2,
$$

which is a contradiction.

LemMA 2.2. Let $x \in S\left(X_{2}\right)$ and let $a=\alpha(x)$, then

(i) There exists a $y \in S\left(X_{2}\right)$ such that $\|y-x\|=\|y+x\|$. Moreover, such $y$ is unique, and the above common value equals $a$.

(ii) Let $p=(y-x) / a$. Then $p \in S\left(X_{2}\right)$ and $\alpha(p)=2 / a$.

(iii) $\alpha(x)=\beta(x)$.

REMARK. In general, these conclusions may not hold if $\operatorname{dim} X>2$.

Proof. (i) It follows directly from Lemma 2.1 and the definition of $\alpha(x)$.

(ii) Let $y$ be as in (i), let

$$
p=\frac{y-x}{a}, \quad q=\frac{y+x}{a},
$$


then $p, q \in S\left(X_{2}\right)$. Consider the triangles determined respectively by $-x, x$, $y$ and $p, q, 0$, it is clear that

$$
a=\frac{\|y-x\|}{\|p\|}=\frac{2\|x\|}{\|p-q\|},
$$

that is,

$$
\|p-q\|=\frac{2}{a}
$$

A similar argument on the triangles determined by $-y, y, x$ and $-p, q, 0$ yields

$$
\|p+q\|=\frac{2}{a}
$$

It follows from (i) that

$$
\alpha(p)=\|p-q\|=\|p+q\|=\frac{2}{a} .
$$

(iii) We need only observe that the $y$ obtained in (i) also satisfies

$$
\|y-x\|=\|y+x\|=\beta(x) .
$$

CoROllary 2.3. $g\left(X_{2}\right)=j\left(X_{2}\right), G\left(X_{2}\right)=J\left(X_{2}\right)$.

ProOF. By Lemma 2.2(iii).

THEOREM 2.4. Let $X$ be a normed linear space, then

$$
g(X) \leq G(X) \leq J(X), \quad g(X) \leq j(X) \leq J(X) .
$$

Proof. It is clear that $g(X)<G(X)$. Let $x \in S(X)$. For any two dimensional subspaces $X_{2}$ containing $X$,

$$
\alpha(x) \leq \alpha_{X_{2}}(x)=\beta_{X_{2}}(x) \leq \beta(x),
$$

where $\alpha_{X_{2}}, \beta_{X_{2}}$ denote the $\alpha$ and $\beta$ for $X_{2}$. Hence $G(X) \leq J(X)$. The second part of the inequality can be obtained similarly.

We remark that there is no inequality between $G(X)$ and $j(X)$ in general (for example, see Theorem 3.1(iii), and Theorem 3.2(ii)).

THEOREM 2.5. Let $X$ be a normed linear, then $1 \leq g(X) \leq \sqrt{2} \leq J(X) \leq 2$ and $g(X) J(X)=2$.

Proof. It follows directly from the definition that $1 \leq g(X)$ and $J(X) \leq 2$. Let $x \in S(X)$ and let $X_{2}$ be a two dimensional subspace containing $x$. Let 
$p \in S\left(X_{2}\right)$ be defined as in Lemma 2.2, then $\alpha_{X_{2}}(x) \cdot \alpha_{X_{2}}(p)=2$. Without loss of generality, we can assume that $\alpha_{X_{2}}(x) \leq \sqrt{2}$, then

$$
\begin{aligned}
& g(X) \leq \alpha(x) \leq \alpha_{X_{2}}(x) \leq \sqrt{2}, \\
& J(X) \geq \beta(p) \geq \beta_{X_{2}}(p)=\alpha_{X_{2}}(p) \geq \sqrt{2} .
\end{aligned}
$$

To prove the last equality, we let $\alpha=g(X), \beta=J(X)$. For any $\varepsilon>0$, let $x, y \in S(X)$ be such that

$$
\|y-x\|=\|y+x\| \leq \alpha+\varepsilon .
$$

Apply Lemma 2.2(iii) to the subspace $X_{2}$ spanned by $x, y$, we can find $p \in$ $S\left(X_{2}\right)$ such that

$$
\frac{2}{\alpha+\varepsilon} \leq \alpha_{X_{2}}(p)=\beta_{X_{2}}(p) \leq \beta .
$$

This implies that $2 \leq \alpha \cdot \beta$. On the other hand, for any $\varepsilon>0$, we can choose $x^{\prime}, y^{\prime} \in S(X)$ such that

$$
\beta-\varepsilon \leq\left\|y^{\prime}-x^{\prime}\right\|=\left\|y^{\prime}+x^{\prime}\right\| .
$$

By applying the same argument as above, we have $\alpha \cdot \beta \leq 2$. This completes the proof that $g(X) \cdot J(X)=2$.

In the rest of this section, we will consider some special two dimensional normed linear spaces. In [3, 4], Gao proved

Proposition 2.6. $S\left(X_{2}\right)$ is affinely homeomorphic to a parallelogram if and only if $g\left(X_{2}\right)=1$. In this case, $j\left(X_{2}\right)=1$ and $G\left(X_{2}\right)=J\left(X_{2}\right)=2$.

Proposition 2.7. If $S\left(X_{2}\right)$ is affinely homeomorphic to a hexagon and has $e$ as one of its vertices, then $g\left(X_{2}\right)=j\left(X_{2}\right)=4 / 3$, and $\beta(e)=G\left(X_{2}\right)=$ $J\left(X_{2}\right)=3 / 2$.

Proposition 2.8. If $S\left(X_{2}\right)$ is affinely homeomorphic to a convex symmetric body in the two dimensional Euclidean space $R_{2}$ which is invariant under $a$ rotation of $45^{\circ}$, then $g(X)=G(X)=j(X)=J(X)=\sqrt{2}$.

REMARK. A circle or an octagon will satisfy the above condition.

Proof. Let $S\left(X_{2}\right)$ be in $R_{2}$ such that it is invariant under a rotation of $45^{\circ}$. Let $x \in S\left(X_{2}\right)$, and let $x_{1}, x_{2}, x_{3}$ be on the arc of $S\left(X_{2}\right)$ in the counterclockwise direction so that the consecutive vectors $x, x_{1}, x_{2}, x_{3}$ form $45^{\circ}$ angles. A rotation of $90^{\circ}$ implies

$$
\left\|x_{2}-x\right\|=\left\|x_{2}+x\right\|
$$


and hence these numbers equal $\alpha(x)$ be Lemma 2.2(i). Also a rotation of $45^{\circ}$ implies that

$$
\alpha\left(x_{3}\right)=\left\|x_{3} \pm x_{1}\right\|=\left\|x_{2} \pm x\right\|=\alpha(x) .
$$

and hence by Lemma $2.2($ ii),

$$
\alpha^{2}(x)=\alpha(x) \alpha\left(x_{3}\right)=2 .
$$

Since this is true for all $x \in S\left(X_{2}\right), g(X)=J(X)=\sqrt{2}$.

It is easy to see that if $X$ is an inner product space, then for any fixed $\lambda>0$,

$x, y \in s(X), \quad\|x+\lambda y\|=\|x-\lambda y\| \Rightarrow\|x+\lambda y\|^{2}=\|x\|^{2}+\lambda^{2}\|y\|^{2}=1+\lambda^{2}$.

In [12] Borwein and Keener asked whether the existence of such $\lambda$ implies that $X$ is an inner product space. We answer this question negatively: Let $X$ be a two dimensional space where $S(X)$ equals the octagon as in the Remark after Proposition 2.8, and let $\lambda=1$. The above condition is satisfied by observing that for any $x, y \in S(X),\|x+y\|=\|x-y\|$ implies that their common value is $\sqrt{2}$.

\section{Special spaces}

Let $l_{p}, L_{p}[0,1]=L_{p}, 1 \leq p \leq \infty$, be defined as in the usual sense. We will evaluate the values of their $\alpha$ and $\beta$ girths and $\alpha$ and $\beta$ perimeters.

TheOREM 3.1. Let $p, q \geq 1$ be such that $1 / p+1 / q=1$.

(i) For $2 \leq p<\infty, g\left(l_{p}\right)=j\left(l_{p}\right)=G\left(l_{p}\right)=2^{1 / p}, J\left(l_{p}\right)=2^{1 / q}$.

(ii) For $1 \leq p<2, g\left(l_{p}\right)=2^{1 / q}, j\left(l_{p}\right)=G\left(l_{p}\right)=J\left(l_{p}\right)=2^{1 / p}$.

(iii) $g\left(l_{\infty}\right)=j\left(l_{\infty}\right)=1, G\left(l_{\infty}\right)=J\left(l_{\infty}\right)=2$.

Proof. (i) We will prove: for $2 \leq p \leq \infty$,

$$
2^{1 / p} \leq g\left(l_{p}\right), \quad j\left(l_{p}\right) \leq 2^{1 / p} \text { and } G\left(l_{p}\right) \leq 2^{1 / p},
$$

then Theorem 2.4 and Theorem 2.5 will imply the result. Recall the Clarkson inequality $[1,2]$ that when $p \geq 2, x, y \in X$,

$$
2\left(\|x\|^{p}+\|y\|^{p}\right) \leq\|x+y\|^{p}+\|x-y\|^{p} \leq 2^{p-1}\left(\|x\|^{p}+\|y\|^{p}\right) .
$$

Hence for $x, y \in S\left(l_{p}\right), p \geq 2$,

$$
2^{2} \leq\|x+y\|^{p}+\|x-y\|^{p} .
$$

This implies that $2^{1 / p} \leq \alpha(x)$. Since $x \in S\left(l_{p}\right)$ is arbitrary, we have $2^{1 / p} \leq$ $g\left(l_{p}\right)$. 
For the second inequality of $(3.1)$, we let $e_{1}=(1,0,0, \ldots)$. For any $y \in$ $\left(y_{1}, \ldots, y_{n}, \ldots\right) \in S\left(l_{p}\right)$, if $y_{1} \geq 0$, then

$$
\left\|y-e_{1}\right\|^{p}=\left(1-y_{1}\right)^{p}+\sum_{i=2}^{\infty}\left|y_{i}\right|^{p} \leq 1+\sum_{i=1}^{\infty}\left|y_{i}\right|^{p}=2 .
$$

Similarly if $y_{1}<0$, then $\left\|y+e_{1}\right\|^{p} \leq 2$. In either case, we have $j\left(l_{p}\right) \leq \beta\left(e_{1}\right) \leq$ $2^{1 / p}$.

To prove $G\left(l_{p}\right) \leq 2^{1 / p}$, we let $x=\left(x_{1}, x_{2}, \ldots\right) \in S\left(l_{p}\right)$. For any $\varepsilon>0$ such that $(1+\varepsilon)^{p}<1+2 p \varepsilon$. There exists $N$ such that $\left|x_{N}\right|<\varepsilon$. Let $y=e_{N}$, then

$$
\|x \pm y\|^{p} \leq 2+2 p \varepsilon .
$$

This implies that

$$
\alpha(x) \leq(2+2 p \varepsilon)^{1 / p} .
$$

Since $\varepsilon$ and $x$ are arbitrary, we have $G\left(l_{p}\right) \leq 2^{1 / p}$.

(ii) We need only show that

$$
2^{1 / p} \leq G\left(l_{p}\right), \quad J\left(l_{p}\right) \leq 2^{1 / p} \text { and } 2^{1 / p} \leq j\left(l_{p}\right) .
$$

The argument is the same as in (i), the corresponding Clarkson inequality we use is: for $1 \leq p<2, x, y \in X$

$$
\|x+y\|^{p}+\|x-y\|^{p} \leq 2\left(\|x\|^{p}+\|y\|^{p}\right) .
$$

(iii) It is clear that $g\left(l_{\infty}\right)=1$. That $j\left(l_{\infty}\right) \leq 1$ follows from the same proof as in (i). To show that $G\left(l_{\infty}\right) \geq 2$, we let $x=(1,1, \ldots)$, then for any $y \in S\left(l_{\infty}\right)$, either $\|x+y\|=2$ or $\|x-y\|=2$. This implies that $\alpha(x)=2$, and hence $G\left(l_{\infty}\right) \geq 2$.

THEOREM 3.2. Let $p, q \geq 1$ be such that $1 / p+1 / q=1$, then

(i) For $2 \leq p<\infty, g\left(L_{p}\right)=G\left(L_{p}\right)=2^{1 / p}, j\left(L_{p}\right)=J\left(L_{p}\right)=2^{1 / q}$.

(ii) For $1 \leq p<2, g\left(L_{p}\right)=G\left(L_{p}\right)=2^{1 / q}, j\left(L_{p}\right)=J\left(L_{p}\right)=2^{1 / p}$.

(iii) $g\left(L_{\infty}\right)=1, j\left(L_{\infty}\right)=G\left(L_{\infty}\right)=J\left(L_{\infty}\right)=2$.

Proof. (i) If suffices to prove the following inequalities as in Theorem 3.1:

$$
2^{1 / p} \leq j\left(L_{p}\right), \quad J\left(L_{p}\right) \leq 2^{1 / q} \text { and } G\left(L_{p}\right) \leq 2^{1 / p} .
$$

Let $x \in S\left(L_{p}\right)$, there exists $\alpha \in[0,1]$ such that

$$
\int_{0}^{\alpha}|x(t)|^{p} d t=\frac{1}{2}
$$

Let $y \in L_{p}$ be defined as

$$
y(t)= \begin{cases}x(t), & 0 \leq t \leq \alpha \\ -x(t), & \alpha<t \leq 1\end{cases}
$$


Then $\|y\|=1$ and

$$
\begin{aligned}
& \|y-x\|^{p}=2^{p} \int_{\alpha}^{1}|x(t)|^{p} d t=2^{p-1}, \\
& \|y+x\|^{p}=2^{p} \int_{\alpha}^{1}|x(t)|^{p} d t=2^{p-1} .
\end{aligned}
$$

It follows from Lemma $2.2(\mathrm{i})$, (iii) that $2^{1 / q} \leq \beta(x)$. Since $x$ is arbitrary, we have $2^{1 / q} \leq j\left(L_{p}\right)$. The second inequality follows from the second part of Clarkson inequality (3.2) in the proof of Theorem 3.1(i).

To prove that $G\left(L_{p}\right) \leq 2^{1 / p}$, we let $x \in S\left(L_{p}\right)$. For any $\varepsilon>0$ such that $(1+\varepsilon)^{p}<1+2 p \varepsilon$, we can find $\delta>0$ with

$$
\int_{0}^{\delta}|x(t)|^{p} d t<\varepsilon
$$

Let $z \in L_{p}$ be defined as

$$
z(t)= \begin{cases}\delta^{-1 / p}, & \text { if } 0<t<\delta \\ 0, & \text { otherwise }\end{cases}
$$

Then

$$
\|z \pm x\|^{p} \leq 2+2 p \varepsilon^{1 / p} .
$$

This implies that $\alpha(x) \leq\left(2+2 p \varepsilon^{1 / p}\right)^{1 / p}$. Since $x$ and $\varepsilon$ are arbitrary, we have $G\left(L_{p}\right) \leq 2^{1 / p}$.

(ii) The proof is similar to the proof of Theorem 3.1(ii).

(iii) It is easy to show that $g\left(L_{\infty}\right)=1$ by considering the two dimensional subspace span by $\chi_{[, 1 / 2]}$ and $\chi_{(1 / 2,0]}$, where $\chi_{A}$ is the indicator function on $A$. To show that $j\left(L_{\infty}\right)=2$, we can find, for any $x \in S\left(L_{\infty}\right)$ and $\varepsilon>0$, and $\alpha \in(0,1)$ such that

$$
\operatorname{ess} \sup \{x(t): t \in[0, \alpha]\}=\operatorname{ess} \sup \{x(t): t \in[\alpha, 1]\}=1-\varepsilon
$$

then proceed to construct the $y$ as in (i). To show that $G\left(L_{\infty}\right)=2$, we can adopt the same proof as in Theorem 3.1(iii).

To conclude this section, we will obtain the values $J(X)$ for the uniformly convex spaces and the uniformly nonsquare spaces.

A normed linear space $X$ is called uniformly convex if for any $0<\varepsilon \leq 2$, there exists $\delta(\varepsilon)>0$ such that for $x, y \in S(X)$ with $\|x-y\| \geq \varepsilon$, then $\|x+y\|<2-2 \delta(\varepsilon)$. Let

$$
\delta_{0}(\varepsilon)=\inf \left\{1-\frac{1}{2}\|x+y\|: x, y \in S(X),\|x-y\| \geq \varepsilon\right\} .
$$

It is clear that $X$ is uniformly convex if and only if $\delta_{0}(\varepsilon)>0$. 
TheOREM 3.3. If $X$ is a normed linear space, then

$$
J(X)=\sup \left\{\varepsilon: \varepsilon<2-2 \delta_{0}(\varepsilon)\right\}
$$

Proof. Let

$$
\varepsilon_{0}=\sup \left\{\varepsilon: \varepsilon<2-2 \delta_{0}(\varepsilon)\right\},
$$

then $0<\varepsilon_{0}<2$. For any $\eta_{0}, \varepsilon>0$ such that $\varepsilon=\varepsilon_{0}+\eta_{0}<2$, we have

$$
2-2 \delta_{0}(\varepsilon) \leq \varepsilon \text {. }
$$

Let $x, y \in S(X)$, then either $\|y-x\| \leq \varepsilon$, or $\|y-x\|>\varepsilon$. In the latter case, we have

$$
\|y+x\| \leq 2-2 \delta_{0}(\varepsilon) \leq \varepsilon .
$$

Hence we conclude that $\beta(x)<\varepsilon$. Since $x$ and $\eta_{0}$ are arbitrary, we have $J(X) \leq \varepsilon_{0}$.

On the other hand, let $\eta_{0}>0$, and let $\varepsilon=\varepsilon_{0}-\eta_{0}$, then there exists $x, y \in S(X)$ such that $\|x-y\|>\varepsilon$, and

$$
1=\left\|\frac{x+y}{2}\right\|<\delta_{0}(\varepsilon)+\eta_{0}
$$

that is

$$
\|x+y\|>2-2 \delta_{0}\left(\varepsilon-2 \eta_{0} .\right.
$$

This implies that

$$
\begin{aligned}
\beta(x) & \geq \min \{\|y-x\|,\|y+x\|\} \\
& \geq \min \left\{\varepsilon, 2-2 \delta_{0}(\varepsilon)-2 \eta_{0}\right\} \\
& \geq \min \left\{\varepsilon, \varepsilon-2 \eta_{0}\right\} \\
& =\varepsilon_{0}-2 \eta_{0} .
\end{aligned}
$$

Since $\eta_{0}$ is arbitrary, we have

$$
J(X) \geq \beta(x) \geq \varepsilon_{0} .
$$

A normed linear space is called uniformly nonsquare [5] if there exists a $\delta>0$ such that $x, y \in S(X)$, either

$$
\left\|\frac{1}{2}(x+y)\right\| \leq 1-\delta \text { or }\left\|\frac{1}{2}(x-y)\right\| \leq 1-\delta .
$$

THEOREM 3.4. $X$ is uniformly nonsquare if and only if $J(X)<2$.

Proof. Suppose $X$ is uniformly nonsquare. Let $\delta$ be as in the definition, then for $x, y \in S(X)$

$$
\min \{\|y-x\|,\|y+x\|\} \leq 2-2 \delta,
$$


and hence $J(X)<2$. Conversely, let $\delta>0$ satisfy $J(X)<2-\delta$. Then the definition of $J(X)$ implies that the above $\delta$ will satisfy the condition in the definition of uniformly nonsquare.

\section{Isomorphism}

Let $X, Y$ be normed linear spaces and let $T: X \rightarrow Y$ be an isomorphism. Following the notation of Schäffer [10], we define $\partial T: S(X) \rightarrow S(Y)$ by

$$
(\partial T)(x)=\frac{T x}{\|T x\|} .
$$

It is clear that $\partial T$ is a bijection. The following lemma is analogous to [10, $5 \mathrm{M}]$.

Lemma 4.1. Let $T: X \rightarrow Y$ be an isomorphism, then for any $x, y y \in S(X)$,

$$
\frac{1}{\|T\| \cdot\left\|T^{-1}\right\|} \leq \frac{\|(\partial T) y-(\partial T) x\|+2}{\|y-x\|+2} \leq\|T\| \cdot\left\|T^{-1}\right\| .
$$

Proof. For any $x, y \in S(X)$

$$
\begin{aligned}
\|(\partial T) y-(\partial T) x\| \leq & \|(\partial T) y-\| T^{-1}\|(T y)\|+\|\| T^{-1}\|(T y)-\| T^{-1}\|(T x)\| \\
& +\|\| T^{-1}\|T x-(\partial T) x\| \\
\leq & \left(\left\|T^{-1}\right\| \cdot\|T\|-1\right)+\|T\| \cdot\left\|T^{-1}\right\| \cdot\|y-x\| \\
& +\left(\left\|T^{-1}\right\| \cdot\|T\|-1\right) .
\end{aligned}
$$

Solving for $\|T\| \cdot\left\|T^{-1}\right\|$ yields the second inequality. The first inequality can be obtained by observing the symmetric role for $(\partial T)^{-1}$.

TheOREM 4.2. Let $T: X \rightarrow Y$ be an isomorphism on the normed linear spaces $X$ and $Y$, then

$$
\frac{1}{\|T\| \cdot\left\|T^{-1}\right\|} \leq \frac{g(Y)+2}{g(X)+2} \leq\|T\| \cdot\left\|T^{-1}\right\| .
$$

The same inequalities also hold if $g$ is replaced by $G, j$ or $J$.

Proof. Let $g(X)=a$. For any $\varepsilon>0$, there exists $x, y \in S(X)$ such that

$$
\max [\|y-x\|,\|y+x\|]<a+\varepsilon .
$$

Then by Lemma 4.1,

$$
\max (\|(\partial T) y \pm(\partial T) x\|)+2 \leq\|T\| \cdot\left\|T^{-1}\right\| \cdot(a+\varepsilon+2) .
$$


This implies that

$$
g(Y)+2 \leq\|T\| \cdot\left\|T^{-1}\right\| \cdot(a+2)
$$

that is

$$
\frac{g(Y)+2}{g(X)+2} \leq\|T\| \cdot\left\|T^{-1}\right\| .
$$

The other inequality can be obtained by interchanging the role of $X, Y$.

The proofs for the other three parameters are identical with this.

Let $X$ be a given normed linear space and let $\widetilde{X}$ be the class of spaces isomorphic to $X$. We define a pseudo metric $\Delta$ on $\widetilde{X}$ as: For $Y, Z \in \widetilde{X}$

$$
\Delta(Y, Z)=\inf \left\{\ln \|T\| \cdot\left\|T^{-1}\right\|: T \text { is an isomorphism from } Y \text { onto } Z\right\} .
$$

Theorem 4.3. Let $p, q \geq 1$ be such that $1 / p+1 / q=1$. If $X$ is a normed linear space such that either

$$
\Delta\left(X, l_{p}\right)<\ln \frac{4}{2^{1 / q}+2}, \quad 2 \leq p<\infty
$$

or

$$
\Delta\left(X, l_{p}\right)<\ln \frac{4}{2^{1 / p}+2}, \quad 1<p \leq 2,
$$

holds, then $X$ is uniformly nonsquare.

Proof. We will consider case (i) only, the second case is similar. Note that Theorem 4.2 implies that

$$
\frac{J(X)+2}{J\left(l_{p}\right)+2} \leq e^{\Delta\left(X, l_{p}\right)} .
$$

In order that $J(X)$ be uniformly nonsquare, it is necessary and sufficient that $J(X)<2$ (Theorem 3.4). This is the case if

$$
e^{\Delta\left(X, l_{p}\right)}\left(J\left(l_{p}\right)+2\right)-2<2
$$

that is

$$
\Delta\left(X, l_{p}\right)<\ln \left(\frac{4}{2^{1 / q}+2}\right)
$$

THEOREM 4.4. The above theorem is also true if $l_{p}$ is replaced by $L_{p}$.

\section{Acknowledgement}

Both authors wish to thank Professor J. J. Schäffer and the referee for some valuable comments in preparing the manuscript. 


\section{References}

[1] J. A. Clarkson, 'Uniformly convex spaces', Trans. Amer. Math. Soc. 40 (1936), 396-414.

[2] M. M. Day, Normed linear spaces (Springer-Verlag, New York-Heidelberg-Berlin, 1973, 2nd ed.).

[3] J. Gao, 'The uniform degree of the unit ball of a Banach space', Nanjing Daxue Xuebao 1 (1982), 14-28 (in Chinese).

[4] J. Gao, 'The extreme value of the uniform degree of the unit ball in a Banach space', Nanjing Daxue Xuebao 1 (1983), 5-12 (in Chinese).

[5] R. C. James, 'Uniformiy non-square spaces', Ann. of Math. 80 (1964), 542-550.

[6] R. C. James and J. J. Schäffer, 'Superreflexivity and the girth of spheres', Israel J. Math. 11 (1972), 398-402.

[7] P. Nyikos and J. J. Schäffer, 'Flat spaces of continuous functions', Studia Math. 42 (1972), 221-229.

[8] J. J. Schäffer, 'On the geometry of spheres in L spaces', Israel J. Math. 10 (1971), 114-120.

[9] J. J. Schäffer, 'On the geometry of spheres of continuous functions,' J. Analyse Math. 26 (1973), 337-389.

[10] J. J. Schäffer, Geometry of spheres in normed spaces (Marcel Dekker, New York, 1976).

[11] J. J. Schäffer and Sundaresen, 'Reflexivity and the girth of spheres, Math. Ann. 184 (1970), 163-168.

[12] J. Borwein and L. Keener, 'The Hausdorff metric and Čebyšev centres', J. Approx. Theory 28 (1980), 366-376.

[13] J. Gao and K. S. Lau, 'On two classes of Banach spaces with normal structure', submitted.

\section{University of Pittsburgh}

Pittsburgh, Pennsylvania

U.S.A. 